\title{
Different distant metastasis patterns based on tumor size could be found in extensive-stage small cell lung cancer patients: a large, population-based SEER study
}

\author{
Jia Li ${ }^{\text {Equal first author, } 1}$, Feng Liu ${ }^{\text {Equal first author, } 1}$, Haining Yu ${ }^{2}$, Chenglong Zhao ${ }^{2}$, Zhenxiang Li $^{3}$, Haiyong Wang $^{\text {Corresp. } 4}$ \\ ${ }^{1}$ Department of Oncology, Longhua Hospital affiliated with Shanghai University of Traditional Chinese Medicine(TCM), ShangHai, China \\ 2 Department of Pathology Oncology, Shandong Cancer Hospital and Institute, Shandong Cancer Hospital affiliated with Shandong University, Shandong \\ Academy of Medical Sciences, Jinan, China \\ 3 Department of Radiation Oncology, Shandong Cancer Hospital and Institute, Shandong Cancer Hospital affiliated with Shandong University, Shandong \\ Academy of Medical Sciences, Jinan, China \\ 4 Department of internal Medicine-Oncology, Shandong Cancer Hospital and Institute, Shandong Cancer Hospital affiliated with Shandong University, \\ Shandong Academy of Medical Sciences, Jinan, China \\ Corresponding Author: Haiyong Wang \\ Email address: wanghaiyong6688@126.com
}

BACKGROUND: Small-cell lung cancer (SCLC) is a malignant cancer with the ability to metastasize quickly. The relationship between tumor size and the distant metastasis patterns of Extensive-Stage Small Cell Lung Cancer (ES-SCLC) has not been reported. OBJECTIVES: The aim of this study was to determine the different distant metastasis patterns as they related to tumor size in ES-SCLC. PATIENTS AND METHODS: We used Surveillance, Epidemiology, and End Results (SEER) population-based data collected from 2010 through 2013 to identify 11058 ES-SCLC patients with definite evidence of distant metastases. Multivariate logistic regression analysis was used to demonstrate the association between tumor size and distant metastasis patterns including bone, liver, brain, and lung metastases. Age, race, sex, and N stage were also selected in the logistic regression model. RESULTS: Subtle differences in metastasis patterns were found among patients based on different tumor sizes. Patients with tumors 3-7 cm have a higher risk of bone metastasis compared with those that have tumors $\leq 3 \mathrm{~cm}(\mathrm{OR} 1.165,95 \% \mathrm{Cl}$ 1.055-1.287 $\square \mathrm{P}=0.003$ ) and patients with tumors $\geq 7 \mathrm{~cm}$ have a higher risk of lung metastasis (OR $1.183,95 \% \mathrm{Cl} 1.039-1.347 \square \mathrm{P}=0.011$ ). In addition, patients with tumors $\geq 7$ $\mathrm{cm}$ had a lower risk of brain metastasis and liver metastasis than patients with tumors $\leq 3$

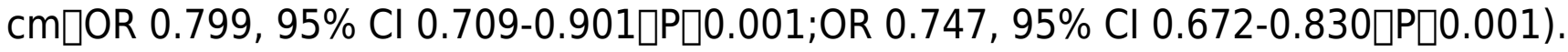
Interestingly, there was no correlation between a larger tumor and a higher risk of metastasis. However, the tumor metastasis pattern did have some correlation with age, gender, race and N-status. CONCLUSION: The pattern of distant metastasis of ES-SCLC is related to the tumor size and the tumor size is indicative of the metastatic site. Larger tumor sizes did not correlate with a higher risk of distant metastasis but the size is related Peer] reviewing PDF | (2019:06:38940:1:1:REVIEW 9 Oct 2019) 
to the pattern of distant metastasis. The study of different distant metastasis patterns based on tumor size and other clinical features (eg. age, race, sex, and N stage) in ESSCLC is clinically valuable. 
1 Title: Different distant metastasis patterns based on tumor size could be found in extensive-stage small cell

2 lung cancer patients: a large, population-based SEER study

3 Author order: Jia Li ${ }^{1 \#}$, Feng Liu ${ }^{1 \#}$,Haining $\mathrm{Yu}^{2}$,Longcheng $\mathrm{Zhao}^{2}$,Zhenxiang $\mathrm{Li}^{3}$, Haiyong Wang ${ }^{4 *}$.

4 Author affiliations:

$5{ }^{1}$ Department of Oncology, Longhua Hospital affiliated with Shanghai University of Traditional Chinese

6 Medicine (TCM), Shanghai 200032, China.

7 2Department of Pathology Oncology, Shandong Cancer Hospital and Institute, Shandong Cancer Hospital

8 affiliated with Shandong University, Shandong Academy of Medical Sciences, Jinan 250117, China.

$9{ }^{3}$ Department of Radiation Oncology, Shandong Cancer Hospital and Institute, Shandong Cancer Hospital

10 affiliated with Shandong University, Shandong Academy of Medical Sciences, Jinan 250117, China.

$11{ }^{4}$ Department of Internal Medicine-Oncology, Shandong Cancer Hospital and Institute, Shandong Cancer

12 Hospital affiliated with Shandong University, Shandong Academy of Medical Sciences, Jinan 250117, China.

\section{*Corresponding authors:}

Haiyong Wang

Department of Internal Medicine-Oncology, Shandong Cancer Hospital and Institute, Shandong Cancer

Hospital affiliated to Shandong University, Shandong Academy of Medical Sciences, Jinan 250117, China.

Tel: 860531-67626332; Fax: 86531-67626332;

E-mail: wanghaiyong6688@126.com

\section{ABSTRACT:}

BACKGROUND: Small-cell lung cancer (SCLC) is a malignant cancer with the ability to metastasize quickly.

The relationship between tumor size and the distant metastasis patterns of Extensive-Stage Small Cell Lung 
22 Cancer (ES-SCLC) has not been reported.

23 OBJECTIVES: The aim of this study was to determine the different distant metastasis patterns as they related to tumor size in ES-SCLC.

PATIENTS AND METHODS: We used Surveillance, Epidemiology, and End Results (SEER) populationbased data collected from 2010 through 2013 to identify 11058 ES-SCLC patients with definite evidence of distant metastases. Multivariate logistic regression analysis was used to demonstrate the association between tumor size and distant metastasis patterns including bone, liver, brain, and lung metastases. Age, race, sex, and $\mathrm{N}$ stage were also selected in the logistic regression model.

RESULTS: Subtle differences in metastasis patterns were found among patients based on different tumor sizes. Patients with tumors 3-7 cm have a higher risk of bone metastasis compared with those that have tumors $\leqslant 3 \mathrm{~cm}(\mathrm{OR} 1.165,95 \%$ CI 1.055-1.287, $\mathrm{P}=0.003)$ and patients with tumors $\geqslant 7 \mathrm{~cm}$ have a higher risk of lung metastasis (OR 1.183, 95\% CI 1.039-1.347, $\mathrm{P}=0.011)$. In addition, patients with tumors $\geqslant 7 \mathrm{~cm}$ had a lower risk of brain metastasis and liver metastasis than patients with tumors $\leqslant 3 \mathrm{~cm}$ (OR $0.799,95 \%$ CI 0.709-0.901, $\mathrm{P}<0.001$; OR $0.747,95 \%$ CI $0.672-0.830, \mathrm{P}<0.001)$. Interestingly, there was no correlation between a larger tumor and a higher risk of metastasis. However, the tumor metastasis pattern did have some correlation with age, gender, race and N-status.

CONCLUSION: The pattern of distant metastasis of ES-SCLC is related to the tumor size and the tumor size is indicative of the metastatic site. Larger tumor sizes did not correlate with a higher risk of distant metastasis but the size is related to the pattern of distant metastasis. The study of different distant metastasis patterns based on tumor size and other clinical features (eg. age, race, sex, and N stage) in ES-SCLC is clinically valuable. 
43

44 Small-cell lung cancer (SCLC) is an extremely aggressive malignancy with approximately 31,000 cases

diagnosed annually in the United States. It comprises $14 \%$ of total lung cancer diagnoses [1]. SCLC is a malignant tumor with high metastatic ability and many metastatic sites, of which bone, brain, liver, and lungs are the most common [2-3]. Many factors affect the metastasis of small cell lung cancer such as tumor size, lymph node involvement, histological subtype, functional status, age, and gender [4-5]. Our current understanding of the relationship between clinically relevant factors and patterns of distant metastasis is limited; few studies have explored the association between tumor size and the sites of distant metastasis [6-8].

Advancements in the treatment of SCLC have lagged behind those for non-small cell lung cancer (NSCLC) and other cancers, especially in the development of molecular profiling and targeted therapies[9]. The ability to predict the risk of distant metastasis with clinically relevant factors in SCLC has important implications for the treatment of this disease.

In this study, the Surveillance, Epidemiology, and End Results (SEER) database was used to analyze the relationship between the size of the tumor and the sites of distant metastasis.

\section{Methods}

\section{Patient selection}

This study was a retrospective study with data obtained from the SEER registry of the US National Cancer Institute [10]. The SEER database catalogues approximately one-quarter of the cancer patients in the United States and is constantly renewing its data. The SEER*Stat software (SEER*Stat 8.3.5) was used to identify and screen for patient data recorded between 2010 and 2013 that would be appropriate for this study (Figure 1). 
64

65

66

67

cytology samples and there was only one primary tumor; 2) the patient was clinically diagnosed as ES-SCLC according to 7\# AJCC staging and had a confirmation of distant metastasis (bone, brain, liver, lung) at the time of initial diagnosis [11]; 3) variables were defined to include age, race, gender, tumor size, and AJCC staging N. Patients with an ambiguous diagnosis or an uncertain site of distant metastasis were excluded. Patients lacking information about variables including age, race, gender, tumor size, AJCC staging N, and metastatic patterns were also excluded.

\section{Ethics statement}

This study was in compliance with the Helsinki Declaration and approved by the Ethics Committee of the Shandong Cancer Hospital. This study was based on the SEER database and did not involve personal privacy information so no informed consent was required.

\section{Statistical analysis}

The variables of age, race, gender, tumor size, N-stage, and metastatic site were included in a multivariate logistic regression analysis to examine the association between clinically relevant factors and specific metastatic patterns. The binomial logistic regression models were used to assess the comparative risk of metastasis. SPSS 22.0 (SPSS, IL, Chicago) was used for data analysis. All statistical tests were bilateral and P< 0.05 was considered statistically significant.

\section{Results}

\section{Patient demographics}

From 2010 to 2013, 11058 ES-SCLC patients were reported in the SEER database; their clinical characteristics are presented in Table $1.41 \%$ of patients were younger than 65 years old, the majority of patients were white (86.4\%), and more than half were male. Moreover, the composition of patients with tumor sizes $\leq 3 \mathrm{~cm}, 3-7$ 
$\mathrm{cm}$, and $\geq 7 \mathrm{~cm}$ accounted for $23.8 \%, 47.6 \%$, and $28.6 \%$ of patients, respectively. According to the AJCC guidelines for the staging of tumor nodule metastasis (TNM), patients with N2 had the highest proportion of metastasis $(57.3 \%)$, while patients with N1 had the lowest proportion with only $6.3 \%$ of the entire cohort, and N3 patients $(24.0 \%)$ were in the middle. In addition, the table shows that a total of $3871(35.0 \%), 2851$ (25.8\%), $4956(44.8 \%)$, and $2275(20.6 \%)$ patients were diagnosed with bone, brain, liver and lung metastases, respectively. The detailed information is presented in Table 1.

All the possible combinations of metastasis patterns are summarized in Table 2 . The results showed that $10.4 \%$ of patients had only bone metastases, $12.7 \%$ had only brain metastases, $17.6 \%$ had only liver metastases, and the proportion of patients with only lung metastases was the lowest, accounting for $6.7 \%$ of the total. The most common two-site metastasis was of the bone and liver $(11.8 \%)$ and the other two-site combination metastases were relatively rare, being those of bone and brain $(2.4 \%)$, bone and lung $(2.0 \%)$, brain and liver $(3.0 \%)$, brain and lung (1.9\%), and liver and lung (3.7\%). The more common three-site combination metastasis was in the bone, brain, and liver (3.1\%) and bone, liver, and lungs (3.5\%). Metastasis to four sites was rare, accounting for $1.2 \%$ of the total and without above four sites(bone,brain,liver,lung) accounted for approximately $18.5 \%$.

\section{Metastasis patterns based on different tumor size}

The metastatic sites were identified as bone metastasis, brain metastasis, liver metastasis, and lung metastasis.

According to the most recent Eighth Edition of the Tumor, Node, and Metastasis (TNM) Classification of Lung Cancer, tumors $\leq 1 \mathrm{~cm}, 1-2 \mathrm{~cm}, 2-3 \mathrm{~cm}, 3-4 \mathrm{~cm}, 4-5 \mathrm{~cm}, 5-7 \mathrm{~cm}$, and $>7 \mathrm{~cm}$ are staged as T1a, T1b, T1c, T2a, T2b, T3, and T4, respectively. In the T stage, $\leq 3 \mathrm{~cm}$ is classified as a T1 tumor, 3-7 cm tumors are classified as T2-T3, and tumors $\geq 7 \mathrm{~cm}$ are classified as T4 tumors [11]. Patients were divided into subgroups according to their tumor sizes of $\leq 3 \mathrm{~cm}, 3-7 \mathrm{~cm}$, and $\geq 7 \mathrm{~cm}$. As shown in Figure $2 \mathrm{~A}$, there are similar 
106

107

108

109

110

111

112

113

114

115

116

117

118

119

120

121

122

123

124

125

126

proportions of tumor metastasis sites among each tumor size group. The liver was the most common site of metastasis and the lungs were the least common site in all ES-SCLCs. The proportion of liver metastasis was lower in the group with tumors of $\geq 7 \mathrm{~cm}(39.8 \%)$ than in the other two groups $(46.8 \%)$. As shown in Figure 2B, patients with tumor sizes of 3-7 cm were more likely to metastasize regardless of the metastatic pattern. In each distant metastatic site, tumors $\leq 3 \mathrm{~cm}$ and $\geq 7 \mathrm{~cm}$ have a small difference in the proportion of distant metastasis.

\section{The association between tumor size and the sites of distant metastasis}

We used multivariate logistic regression analysis to analyze the association between metastatic patterns and clinically relevant factors. Age, race, sex, tumor size, and $\mathrm{N}$ stage were selected in the logistic regression model and were independent risk factors in the multivariable analysis. Age was not an independent risk factor for liver metastasis $(P=0.851)$, and race and gender were not an independent risk factor for lung metastasis ( $P=0.337, P=0.118$, respectively). Compared with patients with tumors $\leq 3 \mathrm{~cm}$, there is a higher risk of bone metastasis in patients with tumors 3-7 $\mathrm{cm}(\mathrm{OR} 1.165,95 \% \mathrm{CI} 1.055-1.287, \mathrm{P}=0.003)$. Patients with tumors $\geqslant 7$ $\mathrm{cm}$ have a higher risk of lung metastasis (OR 1.183, 95\% CI 1.039-1.347, $P=0.011)$ but a lower risk of brain and liver metastases than patients with tumors $\leq 3 \mathrm{~cm} \quad$ (OR 0.799, 95\% CI 0.709-0.901, $P<0.001$; OR $0.747,95 \% \mathrm{CI} 0.672-0.830, P<0.001)$. The relationships between the metastasis pattern and age, and gender, race, and $\mathrm{N}$-status were simultaneously observed. Patients $\geq 65$ years old had a lower risk of bone metastasis and brain metastasis $(P=0.010, P<0.001$, respectively $)$ and a higher risk of lung metastasis $(P<0.001)$. Compared with whites, blacks, and others ethnic patients had a lower risk of bone metastasis and liver metastasis $(P<0.001, P=0.002$ and $P<0.001, P<0.001$, respectively) and black races had a higher risk of brain metastasis $(P=0.004)$. We also found that the higher the $\mathrm{N}$ stage, the higher the risk of bone and liver 
127

128

129

130

131

132

133

134

135

136

137

138

139

140

141

142

143

144

145

146

metastasis $(\mathrm{N} 1, P=0.004 ; \mathrm{N} 2$ and N3, $P<0.001)$ and only patients with $\mathrm{N} 3$ staging had a higher risk of lung metastasis $(P<0.001)$. However, with a higher $\mathrm{N}$ stage, the risk of brain metastasis was reduced $(P<0.001)$

(Table 3).

\section{Discussion}

Small-cell lung cancer (SCLC) is characterized by a rapid doubling time and early, widespread metastasis. The incidence of distant metastasis at the time of the initial diagnosis of SCLC was more than $60 \%$ and the most common metastatic sites were the liver, bone, brain, lung, and adrenal glands [2-3, 12]. The typical treatment of this type of cancer involves small diagnostic biopsies and the rare use of surgical resection, which provides insufficient amounts of tumor tissue for translational research, making it difficult to understand the underlying mechanisms of disease progression and metastasis in SCLC[13-14]. An improved knowledge of the risks factors for different metastatic sites would help to properly classify patients with advanced stages of the disease and may serve as a reference for personalized treatment strategies.

The aim of the study was to better understand the impact of different clinically relevant factors on distant metastasis, particularly the association between tumor size and the exact pattern of distant metastasis. Tumor size is one of the major prognostic factors in the staging system for non-small cell lung cancer (NSCLC) [15]. The prognostic value of the tumor size has been demonstrated in pathological NSCLC and SCLC [3-5, 16-17]. However, the relationship between the tumor size and distant metastasis pattern of SCLC has not been reported.

Bone is the most common site for the distant metastasis of lung cancer. A recent study has shown that patient age $(\mathrm{OR}=1.024, p<0.001)$, the concentration of neuron-specific enolase $(\mathrm{OR}=1.212, p=0.004)$, and histopathological types $(\mathrm{OR}=0.995, p=0.001)$ were the independent risk factors for bone metastasis in 
147 patients with lung cancer [18-19]. Another study has shown that clinical stage, histology, and the

148 clinicopathological characteristics were related to a higher risk of bone metastasis in patients with completely

149 resected non-small-cell lung cancer (NSCLC) [20-22]. The risk factors for bone metastasis in SCLC were not

150 frequently reported and in addition to age, race, gender, and $\mathrm{N}$ stage, the tumor size was also a risk factor for

151 bone metastasis. The higher risk of bone metastasis occurred with the tumors of $3-7 \mathrm{~cm}$. (odds ratio $=1.165, p$

$152=0.003$ ) and not with the larger tumor size of $\geq 7 \mathrm{~cm}$ (odds ratio $=0.965, p=0.531$ ). Previous research has

examined three steps necessary for lung cancer cells to metastasize to bone: i) escape from the primary tumor;

ii) entering the circulation; and iii) colonizing the bone [23]. At the onset of metastasis tumor cells detach from

the cell cluster of the primary tumor and are regulated by a series of cell adhesion factors [24]. We

hypothesized that tumors with a tumor size $>7 \mathrm{~cm}$ would not easily detach from the primary tumor to begin

the subsequent metastasis. However, the specific molecular mechanisms by which lung cancer cells

metastasize to bone still requires further research and exploration. Other research reports that lung cancer is

more prone to bone metastases because of the microenvironment of the bone that is affected by the bone

matrix, the immune system cells, and the same cancer cells [25]. At the time of the initial diagnosis,

approximately $20 \%$ of patients with SCLC have detectable brain metastases [26], which is roughly in line with

Classification of Lung Cancer, a larger tumor indicates a higher T stage [11]. A study showed that high T stage,

high neutrophil-to-lymphocyte ratio, early thoracic radiotherapy, and fewer chemotherapy cycles were risk

factors for brain metastases [27]. Our results were inconsistent with those studies and found that patients with 
168

169

170

171

172

173

174

175

176

177

178

179

180

181

182

odds ratio $=0.729, p<0.001 ; \mathrm{N} 3$, odds ratio $=0.671, p<0.001)$. SCLC is a tumor that develops brain metastasis very early, typically because small cell lung cancer originates from pulmonary neuroendocrine cells and other potential candidate cells, such as alveolar type 2 cells [28]. There is also ample opportunity for the seed cells to find a receptive environment. [29]. It is possible that chemokines and adhesion molecules play an important role in lowering the risk of brain metastasis with larger tumor sizes and higher $\mathrm{N}$ stages [30]. Other studies have reported that the cumulative years of pack smoking is associated with a greater velocity in brain metastasis [31]. Due to the limitations of the SEER database specific information on smoking status was not collected. This study did not analyze the relationship between smoking status and brain metastasis.

The liver was the most prevalent site of metastasis (61.9\%) and liver metastasis was the most common site of hematogenous metastasis in ES-SCLC [32]. Some studies have demonstrated that liver metastasis may not be associated with the advancement of TNM staging [33]. We analyzed the risk factors for liver metastasis from ES-SCLC patients using race, sex, tumor size, and $\mathrm{N}$ stage as independent risk factors in the multivariable analysis. The risk of liver metastasis was lower when the tumor was $\geq 7 \mathrm{~cm}$ (odds ratio $=0.747, p<0.001$ ).

Additional research is necessary to identify why patients with tumors $\geq 7 \mathrm{~cm}$ are not prone to develop distance metastasis, especially to the liver. For lung metastasis, we found that age, tumor size, and $\mathrm{N}$ stage were independent risk factors and that it was more likely for lung metastasis to occur when the tumor was $\geq 7 \mathrm{~cm}$

(odds ratio $=1.183, p=0.011)$ and the $\mathrm{N}$ stage was higher $(\mathrm{N} 3$, odds ratio $=1.501, p<0.001)$. It is possible that the larger tumor size is associated with a higher probability of lymph node metastasis and local disease extension (eg. main stem bronchus involvement, visceral pleura invasion, chest wall invasion) [34]. Finally, we observed the relationship between the metastasis pattern and age, gender, race and N-status. Patients $\geq 65$ 
188

years old had a lower risk of bone and brain metastasis $(P=0.010, P<0.001$, respectively $)$ and a higher risk of lung metastasis $(P<0.001)$. Ethnic patients had lower risk of bone metastasis and liver metastasis when compared with whites, blacks, and others $(P<0.001, P=0.002$ and $P<0.001, P<0.001$, respectively) and black races had a higher risk of brain metastases $(P=0.004)$. The correlation between these clinical factors and distant metastatic sites may involve differences in population characteristics and further exploration is warranted. We also found that higher $\mathrm{N}$ stages $(\mathrm{N} 1, P=0.004$; N2 and N3, $P<0.001)$ correlated with a higher risk of bone and liver metastasis. This is consistent with current reports that the volume and number of metastatic lymph nodes are closely related to the site of metastasis [35].

There are some limitations to this study. First, it is not possible to assess the impact of chemotherapy, radiation therapy, or smoking status on metastasis because this information is missing from the SEER database. Secondly, the aim of this study was to determine the different distant metastasis patterns based on tumor size in ES-SCLC and we only analyzed the relationship between tumor size and the distant metastatic patterns. When extracting data from the SEER database, we did not extract the corresponding information about survival rates. Thirdly, this study is a non-randomized study and although our sample size is large, there are inherent defects in any retrospective study. Fourth, the sites of metastasis that were analyzed were limited to the bone, lungs, liver, and brain. Although the common sites of SCLC metastasis are bone, liver, lungs, and brain $[2-3,12]$, metastasis and the combined metastasis of the adrenal gland or other metastatic sites may occur in ES-SCLC patients. Fifth, the tumor size of small cell lung cancer is relatively difficult to measure, so we only selected data with a clear tumor size from the SEER database and any data with an unclear tumor size was not included in this study.

CONCLUSION: The pattern of distant metastasis of ES-SCLC is related to the tumor size and the tumor size 
209 is indicative of the metastatic site. Larger tumor sizes did not correlate with a higher risk of distant metastasis

210 but the size is related to the pattern of distant metastasis. The study of different distant metastasis patterns

211 based on tumor size and other clinical features (eg. age, race, sex, and N stage) in ES-SCLC is clinically

212 valuable.

213 Acknowledgments: We would like to thank the staff of the National Cancer Institute and their colleagues

214 across the United States and those at Information Management Services, Inc., who have been involved with the

215 Surveillance, Epidemiology and End Results (SEER) Program.

\section{References}

217 1. Tarver T.2014.American Cancer Society Cancer Facts \& Figures.Consumer Health Internet 16(3), 366-367 218 (2012).

219 2. Nakazawa, K., Kurishima, K., Tamura, T., Kagohashi, K., Ishikawa, H., Satoh, H., and Hizawa, N. 2012.

220 Specific organ metastases and survival in small cell lung cancer. Oncology Letters 4:617-620.

221 DOI:10.3892/ol.2012.792.

222 3. Cai, H., Wang, H., Li, Z., Lin, J., and Yu, J. 2018. The prognostic analysis of different metastatic patterns in 223 extensive-stage small-cell lung cancer patients: a large population-based study. Future Oncology 14:1397-1407. 224 DOI:10.2217/fon-2017-0706.

225 4. Tas, F., Aydiner, A., Topuz, E., Camlica, H., Saip, P., and Eralp, Y. 1999. Factors influencing the 226 distribution of metastases and survival in extensive disease small cell lung cancer. ACTA ONCOLOGICA 227 38:1011-1015. DOI:10.1080/028418699432275.

228 5. Riihimaki, M., Hemminki, A., Fallah, M., Thomsen, H., Sundquist, K., Sundquist, J., and Hemminki, K. 229 2014. Metastatic sites and survival in lung cancer. LUNG CANCER 86:78-84. 230 DOI:10.1016/j.lungcan.2014.07.020.

231 6. Wang, H., Han, X., Guo, J., and Wang, Z. 2018.Characteristics and survival difference of clinical tumor size 2320 extensive-stage small cell lung cancer with different metastasis pattern. Journal of Thoracic Disease 
233

234

235

236

237

238

239

240

241

242

243

244

245

246

247

248

249

250

251

252

253

254

255

256

257

258

259

10:5414-5420.DOI: $10.21037 /$ jtd.2018.09.11.

7. Milovanovic, I.S., Stjepanovic, M., and Mitrovic, D. 2017. Distribution patterns of the metastases of the lung carcinoma in relation to histological type of the primary tumor: An autopsy study. Annals of Thoracic Medicine 12:191-198. DOI:10.4103/atm.ATM_276_16.

8. Wang, L., Dou, X., Liu, T., Lu, W., Ma, Y., and Yang, Y. 2018. Tumor size and lymph node metastasis are prognostic markers of small cell lung cancer in a Chinese population. Medicine (Baltimore) 97:e11712. DOI:10.1097/MD.0000000000011712.

9. Byers, L.A., and Rudin, C.M. 2015. Small cell lung cancer: where do we go from here? CANCER 121:664672. DOI:10.1002/cncr.29098.

10. Surveillance: Epidemiology: and End Results (SEER) Program. SEER*Stat Database: Incidence - SEER 18 Regs Research Data +Hurricane Katrina Impacted Louisiana Cases: Nov 2016 Sub (1973-2014 varying) Linked To County Attributes - Total U.S.:1969-2015 Counties: National Cancer Institute: DCCPS: Surveillance Research Program: released April 2017: based on the November 2016 submission. www.seer.cancer.gov.

11. Rami-Porta, R., Asamura, H., Travis, W.D., and Rusch, V.W. 2017. Lung cancer - major changes in the American Joint Committee on Cancer eighth edition cancer staging manual. CA Cancer J Clin 67:138-155. DOI:10.3322/caac.21390.

12. Singh, N., Madan, K., Aggarwal, A.N., and Das, A. 2013. Symptomatic large bilateral adrenal metastases at presentation in small-cell lung cancer: a case report and review of the literature. Journal of Thoracic Disease 5:E83-E86. DOI:10.3978/j.issn.2072-1439.2011.09.05.

13. Qin, A., and Kalemkerian, G.P. 2018. Treatment Options for Relapsed Small-Cell Lung Cancer: What Progress Have We Made? Journal of Oncology Practice 14:369-370. DOI:10.1200/JOP.18.00278.

14. Oberndorfer, F., and Mullauer, L. 2018. Molecular pathology of lung cancer: current status and perspectives. CURRENT OPINION IN ONCOLOGY 30:69-76. DOI:10.1097/CCO.0000000000000429.

15. Giroux, D.J., Van Schil, P., Asamura, H., Rami-Porta, R., Chansky, K., Crowley, J.J., Rusch, V.W., and Kernstine, K. 2018. The IASLC Lung Cancer Staging Project: A Renewed Call to Participation. Journal of Thoracic Oncology 13:801-809. DOI:10.1016/j.jtho.2018.02.012. 
260

261

262

263

264

265

266

267

268

269

270

271

272

273

274

275

276

277

278

279

280

281

282

283

284

285

286

16. Zhang, J., Gold, K.A., Lin, H.Y., Swisher, S.G., Xing, Y., Lee, J.J., Kim, E.S., and William, W.J. 2015. Relationship between tumor size and survival in non-small-cell lung cancer (NSCLC): an analysis of the surveillance, epidemiology, and end results (SEER) registry. Journal of Thoracic Oncology 10:682-690. DOI:10.1097/JTO.0000000000000456.

17. Zhang, Y., Sun, Y., and Chen, H. 2016. Effect of tumor size on prognosis of node-negative lung cancer with sufficient lymph node examination and no disease extension. Onco Targets Ther 9:649653.DOI:10.2147/OTT.S98509.

18. Zhou, Y., Chen, W.Z., Peng, A.F., Tong, W.L., Liu, J.M., and Liu, Z.L. 2017. Neuron-specific enolase, histopathological types, and age as risk factors for bone metastases in lung cancer. Tumour Biol 39:1393375470. DOI:10.1177/1010428317714194.

19. Niu, Y.J., Wen, Y.T., Shen, W.W., Deng, L., Liu, L.L., and Zhang, H.L. 2014. Risk factors for bone metastasis in patients with primary lung cancer: study protocol for a systematic review. BMJ Open 4:e5202. DOI:10.1136/bmjopen-2014-005202.

20. Wang, H., Zhang, Y., Zhu, H., and Yu, J. 2017. Risk factors for bone metastasis in completely resected non-small-cell lung cancer. Future Oncology 13:695-704. DOI:10.2217/fon-2016-0237.

21. Oliveira, M.B., Mello, F.C., and Paschoal, M.E. 2016. The relationship between lung cancer histology and the clinicopathological characteristics of bone metastases. LUNG CANCER 96:19-24. DOI:10.1016/j.lungcan.2016.03.014.

22. Shabani, M., Binesh, F., Behniafard, N., Nasiri, F., and Shamsi, F. 2014. Clinicopathologic characteristics and survival of patients with bone metastasis in Yazd, Iran: a cross-sectional retrospective study. Medicine (Baltimore) 93:e317. DOI:10.1097/MD.0000000000000317.

23. Luo, Q., Xu, Z., Wang, L., Ruan, M., and Jin, G. 2016. Progress in the research on the mechanism of bone metastasis in lung cancer. Mol Clin Oncol 5:227-235. DOI:10.3892/mco.2016.917.

24. Perl, A.K., Wilgenbus, P., Dahl, U., Semb, H., and Christofori, G. 1998. A causal role for E-cadherin in the transition from adenoma to carcinoma. NATURE 392:190-193. DOI:10.1038/32433.

25. Roato, I. 2014. Bone metastases: When and how lung cancer interacts with bone. World J Clin Oncol 5:149-155. DOI:10.5306/wjco.v5.i2.149. 
287

288

289

290

291

292

293

294

295

296

297

298

299

300

301

302

303

304

305

306

307

308

309

310

311

312

313

26. Eze, C., Roengvoraphoj, O., Niyazi, M., Hildebrandt, G., Fietkau, R., Belka, C., and Manapov, F. 2017.

Treatment Response and Prophylactic Cranial Irradiation Are Prognostic Factors in a Real-life Limited-disease

Small-cell Lung Cancer Patient Cohort Comprehensively Staged With Cranial Magnetic Resonance Imaging. Clinical Lung Cancer 18:e243-e249. DOI:10.1016/j.cllc.2016.11.005.

27. Zheng, Y., Wang, L., Zhao, W., Dou, Y., Lv, W., Yang, H., Sun, Y., and Xing, L. 2018. Risk factors for brain metastasis in patients with small cell lung cancer without prophylactic cranial irradiation. STRAHLENTHERAPIE UND ONKOLOGIE 194:1152-1162. DOI:10.1007/s00066-018-1362-7.

28. Bunn, P.J., Minna, J.D., Augustyn, A., Gazdar, A.F., Ouadah, Y., Krasnow, M.A., Berns, A., Brambilla, E., Rekhtman, N., Massion, P.P., Niederst, M., Peifer, M., Yokota, J., Govindan, R., Poirier, J.T., Byers, L.A., Wynes, M.W., McFadden, D.G., MacPherson, D., Hann, C.L., Farago, A.F., Dive, C., Teicher, B.A., Peacock, C.D., Johnson, J.E., Cobb, M.H., Wendel, H.G., Spigel, D., Sage, J., Yang, P., Pietanza, M.C., Krug, L.M., Heymach, J., Ujhazy, P., Zhou, C., Goto, K., Dowlati, A., Christensen, C.L., Park, K., Einhorn, L.H., Edelman, M.J., Giaccone, G., Gerber, D.E., Salgia, R., Owonikoko, T., Malik, S., Karachaliou, N., Gandara, D.R., Slotman, B.J., Blackhall, F., Goss, G., Thomas, R., Rudin, C.M., and Hirsch, F.R. 2016. Small Cell Lung Cancer: Can Recent Advances in Biology and Molecular Biology Be Translated into Improved Outcomes? Journal of Thoracic Oncology 11:453-474. DOI:10.1016/j.jtho.2016.01.012.

29. Lukas, R.V., Gondi, V., Kamson, D.O., Kumthekar, P., and Salgia, R. 2017. State-of-the-art considerations in small cell lung cancer brain metastases. Oncotarget 8:71223-71233. DOI:10.18632/oncotarget.19333.

30. Takano, K., Kinoshita, M., Takagaki, M., Sakai, M., Tateishi, S., Achiha, T., Hirayama, R., Nishino, K., Uchida, J., Kumagai, T., Okami, J., Kawaguchi, A., Hashimoto, N., Nakanishi, K., Imamura, F., Higashiyama, M., and Yoshimine, T. 2016. Different spatial distributions of brain metastases from lung cancer by histological subtype and mutation status of epidermal growth factor receptor. Neuro Oncol 18:716-724. DOI:10.1093/neuonc/nov266.

31. Shenker, R.F., McTyre, E.R., Ruiz, J., Weaver, K.E., Cramer, C., Alphonse-Sullivan, N.K., Farris, M., Petty, W.J., Bonomi, M.R., Watabe, K., Laxton, A.W., Tatter, S.B., Warren, G.W., and Chan, M.D. 2017. The Effects of smoking status and smoking history on patients with brain metastases from lung cancer. Cancer Med 6:944-952. DOI:10.1002/cam4.1058. 
314 32. Ren, Y., Dai, C., Zheng, H., Zhou, F., She, Y., Jiang, G., Fei, K., Yang, P., Xie, D., and Chen, C. 2016.

315 Prognostic effect of liver metastasis in lung cancer patients with distant metastasis. Oncotarget 7:53245-53253.

316 DOI:10.18632/oncotarget.10644.

317 33. Kagohashi, K., Satoh, H., Ishikawa, H., Ohtsuka, M., and Sekizawa, K. 2003. Liver metastasis at the time 318 of initial diagnosis of lung cancer. MEDICAL ONCOLOGY 20:25-28. DOI:10.1385/MO:20:1:25.

319 34. Chen, C., Chen, Z., Cao, H., Yan, J., Wang, Z., Le H, Weng, J., and Zhang, Y. 2018. A retrospective 320 clinicopathological study of lung adenocarcinoma: Total tumor size can predict subtypes and lymph node 321 involvement. Clin Imaging 47:52-56. DOI:10.1016/j.clinimag.2017.08.009.

322 35. Zhang, Y., Sun, Y., and Chen, H. 2016. Effect of tumor size on prognosis of node-negative lung cancer 323 with sufficient lymph node examination and no disease extension. Onco Targets Ther 9:649-653. 324 DOI:10.2147/OTT.S98509.

325 Figure legends

326

327

Figure 1. The percentage of distant metastasis sites. A. The percentage of distant metastasis sites based on different tumor sizes. B. The percentage of tumor sizes based on different distant metastasis sites.

Figure 2. The flow chart of data selection in this study.

Table legends

Table 1: Characteristics of extensive-stage small cell lung cancer from SEER Database from 2010-2013

Table 2: Frequencies of combination metastasis sites in ES-SCLC patients.

Table 3: Multivariate logistic regression analysis was used to evaluate the relationship between tumor size and distant metastasis sites 
Figure 1

The flow chart of data selection in this study .
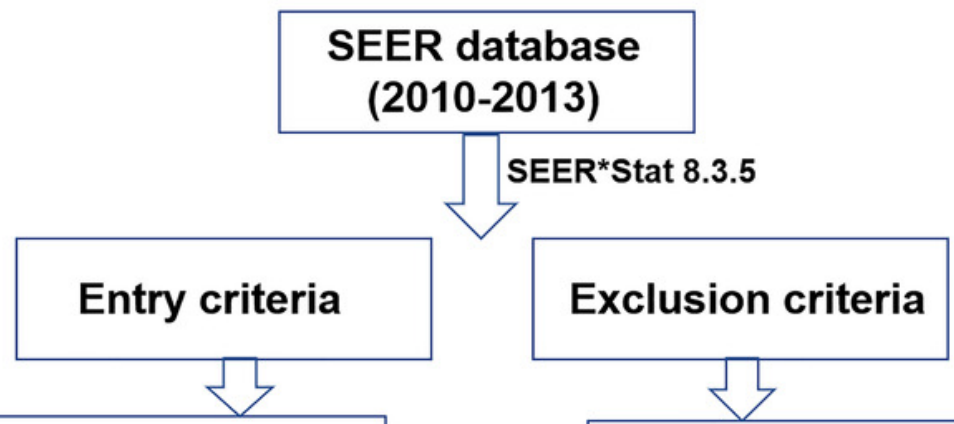

- Pathological confirmation

- Only one primary tumor

- Definite distant metastasis (Bone, Brain, Liver, Lung)

- Definite age, race, sex, AJCC staging $N$

- Definite tumor size

- Ambiguous diagnosis

- The location of metastasis is not clear

- Lack of specific information on age, race, sex, AJCC staging $\mathrm{N}$

- Lack of specific information on tumor size

- Other variables with ambiguous information

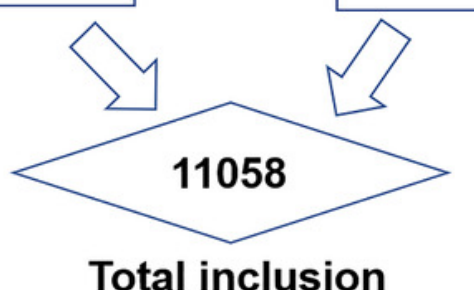


Figure 2

The percentage of distant metastasis sites.

A. The percentage of distant metastasis sites based on different tumor sizes. B. The percentage of tumor sizes based on different distant metastasis sites.
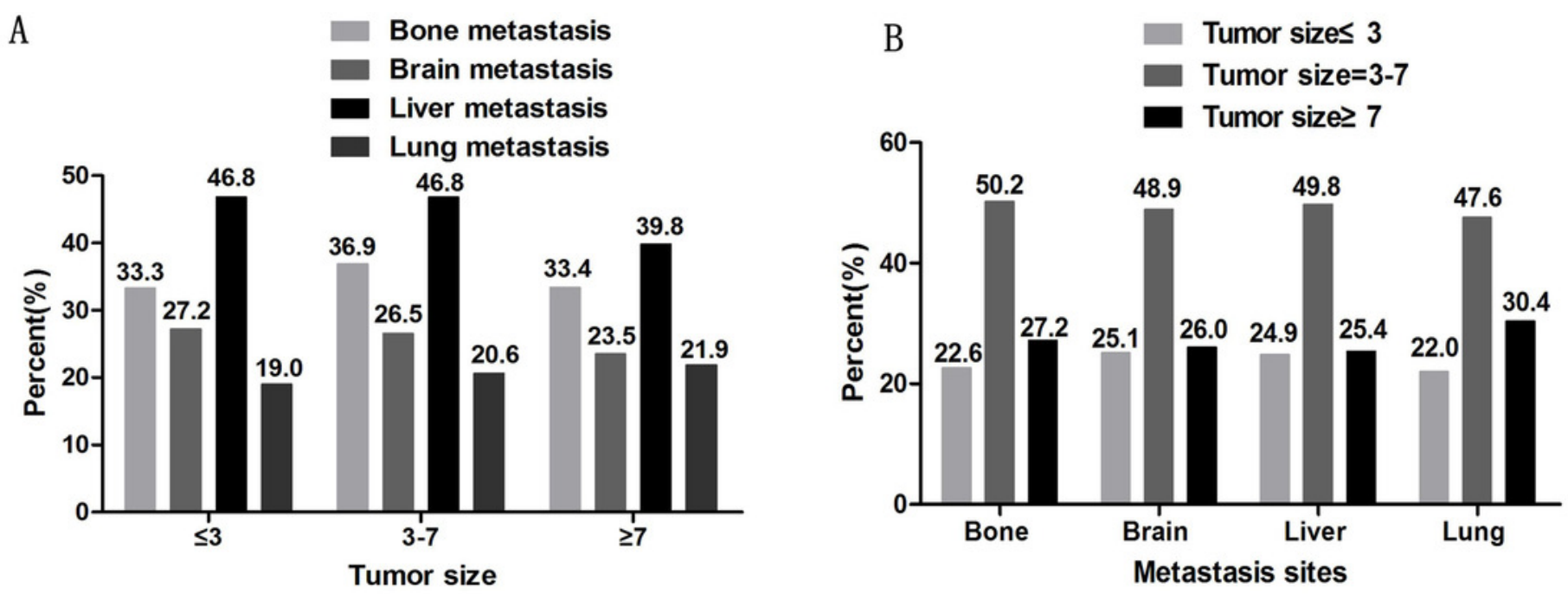
Table $\mathbf{1}$ (on next page)

Characteristics of extensive-stage small cell lung cancer from SEER Database from 2010-2013. 


\begin{tabular}{|c|c|c|}
\hline Variables & Number & $\%$ \\
\hline \multicolumn{3}{|l|}{ Age(years) } \\
\hline$<65$ & 4536 & 41.0 \\
\hline$\geq 65$ & 6522 & 59.0 \\
\hline \multicolumn{3}{|l|}{ Race } \\
\hline White & 9555 & 86.4 \\
\hline Black & 1057 & 9.6 \\
\hline Others & 446 & 4.0 \\
\hline \multicolumn{3}{|l|}{ Sex } \\
\hline Female & 5288 & 47.8 \\
\hline Male & 5770 & 52.2 \\
\hline \multicolumn{3}{|c|}{ Tumor size(cm) } \\
\hline$\leq 3$ & 2630 & 23.8 \\
\hline $3-7$ & 5269 & 47.6 \\
\hline$\geq 7$ & 3159 & 28.6 \\
\hline \multicolumn{3}{|l|}{ N stage } \\
\hline No & 1375 & 12.4 \\
\hline N1 & 693 & 6.3 \\
\hline $\mathrm{N} 2$ & 6341 & 57.3 \\
\hline N3 & 2649 & 24.0 \\
\hline \multicolumn{3}{|c|}{ Bone metastasis } \\
\hline Yes & 3871 & 35.0 \\
\hline No & 7187 & 65.0 \\
\hline \multicolumn{3}{|c|}{ Brain metastasis } \\
\hline Yes & 2851 & 25.8 \\
\hline No & 8207 & 74.2 \\
\hline \multicolumn{3}{|c|}{ Liver metastasis } \\
\hline Yes & 4956 & 44.8 \\
\hline No & 6102 & 55.2 \\
\hline \multicolumn{3}{|c|}{ Lung metastasis } \\
\hline Yes & 2275 & 20.6 \\
\hline No & 8783 & 79.4 \\
\hline
\end{tabular}


Table 2 (on next page)

Frequencies of combination metastasis sites in ES-SCLC patients. 
1 Table 2

2

\begin{tabular}{lcc}
\hline Metastasis & Number & \% \\
\hline Only one site & 1147 & 10.4 \\
Bone & 1401 & 12.7 \\
Brain & 1941 & 17.6 \\
Liver & 741 & 6.7 \\
Lung & & \\
Two sites & 263 & 2.4 \\
Bone+Brain & 1309 & 11.8 \\
Bone+Liver & 224 & 2.0 \\
Bone+Lung & 332 & 3.0 \\
Brain+Liver & 212 & 1.9 \\
Brain+Lung & 406 & 3.7 \\
Liver+Lung & & \\
Three sites & 341 & 3.1 \\
Bone+Brain+Liver & 65 & 0.6 \\
Bone+Brain+Lung & 390 & 3.5 \\
Bone+Liver+Lung & 105 & 0.9 \\
Brain+Liver+Lung & & 1.2 \\
Four sites & 132 & \\
Bone+Brain+Liver+Lung & & \\
Others & 2049 & \\
Without(bone,brain,liver,lung) & &
\end{tabular}




\section{Table 3(on next page)}

Multivariate logistic regression analysis was used to evaluate the relationship between tumor size and distant metastasis sites. 
$1 \quad$ Table 3

\begin{tabular}{|c|c|c|c|c|c|c|c|c|}
\hline \multirow[t]{2}{*}{ Variables } & \multicolumn{2}{|l|}{ Bone metastasis } & \multicolumn{2}{|l|}{ Brain metastasis } & \multicolumn{2}{|l|}{ Liver metastasis } & \multicolumn{2}{|l|}{ Lung metastasis } \\
\hline & OR $(95 \% C I)$ & $P$ & OR $(95 \% C I)$ & $P$ & OR $(95 \% C I)$ & $P$ & OR $(95 \% C I)$ & $P$ \\
\hline Age(years) & & 0.010 & & $<0.001$ & & 0.851 & & $<0.001$ \\
\hline$<65$ & Reference & & Reference & & Reference & & Reference & \\
\hline$\geq 65$ & $0.899(0.830-0.975)$ & 0.010 & $0.703(0.644-0.767)$ & $<0.001$ & $0.993(0.919-1.072)$ & 0.851 & $1.209(1.099-1.330)$ & $<0.001$ \\
\hline Race & & $<0.001$ & & 0.005 & & $<0.001$ & & 0.337 \\
\hline White & Reference & & Reference & & Reference & & Reference & \\
\hline Black & $0.737(0.641-0.848)$ & $<0.001$ & $1.233(1.071-1.419)$ & 0.004 & $0.713(0.625-0.813)$ & $<0.001$ & $1.119(0.959-1.306)$ & 0.152 \\
\hline Others & $0.711(0.576-0.878)$ & 0.002 & $1.196(0.966-1.481)$ & 0.100 & $0.690(0.566-0.841)$ & $<0.001$ & $1.055(0.837-1.329)$ & 0.651 \\
\hline Sex & & $<0.001$ & & 0.001 & & 0.001 & & 0.118 \\
\hline Female & Reference & & Reference & & Reference & & Reference & \\
\hline Male & $1.282(1.184-1.388)$ & $<0.001$ & $1.152(1.057-1.256)$ & $<0.001$ & $1.152(1.068-1.243)$ & 0.001 & $1.077(0.981-1.182)$ & 0.118 \\
\hline Tumor & & $<0.001$ & & $<0.001$ & & $<0.001$ & & 0.040 \\
\hline \multicolumn{9}{|l|}{$\operatorname{Size}(\mathrm{cm})$} \\
\hline$\leq 3$ & Reference & & Reference & & Reference & & Reference & \\
\hline $3-7$ & $1.165(1.055-1.287)$ & 0.003 & $0.968(0.871-1.077)$ & 0.553 & $0.994(0.904-1.092)$ & 0.894 & $1.097(0.975-1.235)$ & 0.125 \\
\hline$\geq 7$ & $0.965(0.863-1.079)$ & 0.531 & $0.799(0.709-0.901)$ & $<0.001$ & $0.747(0.672-0.830)$ & $<0.001$ & $1.183(1.039-1.347)$ & 0.011 \\
\hline N stage & & $<0.001$ & & $<0.001$ & & $<0.001$ & & $<0.001$ \\
\hline No & Reference & & Reference & & Reference & & Reference & \\
\hline N1 & $1.379(1.128-1.687)$ & 0.002 & $1.022(0.839-1.245)$ & 0.829 & $1.346(1.117-1.621)$ & 0.002 & $1.155(0.916-1.458)$ & 0.223 \\
\hline $\mathrm{N} 2$ & $1.570(1.375-1.793)$ & $<0.001$ & $0.729(0.642-0.829)$ & $<0.001$ & $1.500(1.330-1.692)$ & $<0.001$ & $1.134(0.975-1.320)$ & 0.104 \\
\hline N3 & $2.060(1.782-2.382)$ & $<0.001$ & $0.671(0.580-0.777)$ & $<0.001$ & $1.280(1.119-1.465)$ & $<0.001$ & $1.501(1.273-1.770)$ & $<0.001$ \\
\hline \multicolumn{9}{|l|}{2} \\
\hline \multicolumn{9}{|l|}{3} \\
\hline \multicolumn{9}{|l|}{4} \\
\hline \multicolumn{9}{|l|}{5} \\
\hline \multicolumn{9}{|l|}{6} \\
\hline \multicolumn{9}{|l|}{7} \\
\hline \multicolumn{9}{|l|}{8} \\
\hline \multicolumn{9}{|l|}{9} \\
\hline 10 & & & & & & & & \\
\hline
\end{tabular}

